\title{
Application of Gas-Kinetic Scheme with Kinetic Boundary Conditions in Hypersonic Flow
}

\author{
Qibing $\mathrm{Li}^{*}$ and Song $\mathrm{Fu}^{\dagger}$ \\ Tsinghua University, 100084 Beijing, People's Republic of China \\ and \\ Kun $\mathrm{Xu}^{\text {* }}$ \\ Hong Kong University of Science and Technology, Hong Kong, People's Republic of China
}

\begin{abstract}
This work focuses on the application of the gas-kinetic scheme based on the Bhatnagar-Gross-Krook particle collision model in the hypersonic flow simulations. Kinetic boundary conditions with different accommodation coefficients are constructed and implemented in the scheme. The numerical study is based on a laminar shock-wave/boundary-layer interaction in a Mach 10 flow passing through a hollow cylinder flare model. The grid-independent numerical results agree well with experimental measurements. The utilization of the kinetic slip boundary condition is necessary to improve the agreement between the current results, experimental measurements, as well as direct simulation Monte Carlo solutions. The effect of the variation of the accommodation coefficient on the flow solutions is quantitatively evaluated.
\end{abstract}

\section{Introduction}

$\mathbf{R}$ ECENTLY hypersonic flow relating to the boundary layer has been actively studied both numerically and experimentally. For example, the shock-wave/boundary-layer interaction triggers flow separation and reattachment, which could significantly increase the heat flux near the reattachment point. Because of the complex flow physics, it remains a challenge to capture accurately wave interactions with a viscous flow solver. There has been much research effort at validating the Navier-Stokes (NS) and direct simulation Monte Carlo (DSMC) based methods. ${ }^{1-8}$

Based on the gas-kinetic Bhatnagar-Gross-Krook (BGK) model, an accurate Navier-Stokes flow solver has been developed in the past few years. ${ }^{9}$ Two favorable features of this approach are the positivity preserving property and the satisfaction of the entropy condition, both of which are important for any scheme in the study of hypersonic viscous flow. Hence, the gas-kinetic BGK scheme yields reliable numerical results for viscous flows involving complicated wave structures, such as the high-speed mixing layer, ${ }^{10-12}$ hypersonic viscous flow, ${ }^{13}$ and flow with interface. ${ }^{14}$ Additionally, as the flux at the cell interface is calculated through the particle distribution function, it can introduce a slip condition to simulate directly nonequilibrium flow. ${ }^{15,16}$ Thus, the scheme can be matched theoretically with the DSMC method in the near-continuum regime.

In the present paper, the gas-kinetic BGK scheme is applied to the study of the strong shock-wave/boundary-layer interaction around a hollow cylinder flare. The flow studied experimentally in the $\mathrm{R} 5 \mathrm{Ch}$ blowdown hypersonic wind tunnel at ONERA in Toulouse, France, is axisymmetric and laminar. Many comparisons have been made between numerical results with experimental data. ${ }^{1,2,4,7,17}$ Although the Knudsen number is relatively small in this case, the comparative studies show that the thermodynamic nonequilibrium (rarefaction) gas effects are substantial and the implementation of the slip boundary condition is needed to improve the numerical results. In

Received 21 October 2004; revision received 22 April 2005; accepted for publication 19 June 2005. Copyright (C) 2005 by the American Institute of Aeronautics and Astronautics, Inc. All rights reserved. Copies of this paper may be made for personal or internal use, on condition that the copier pay the $\$ 10.00$ per-copy fee to the Copyright Clearance Center, Inc., 222 Rosewood Drive, Danvers, MA 01923; include the code 0001-1452/05 \$10.00 in correspondence with the CCC.

*Lecturer, Department of Engineering Mechanics; lqb@tsinghua.edu.cn.

${ }^{\dagger}$ Professor, Department of Engineering Mechanics. Senior Member AIAA.

${ }^{\ddagger}$ Professor, Mathematics Department, Clear Water Bay, Kowloon. Member AIAA. the present work, we use the BGK scheme to study this problem and evaluate the slip boundary condition in the numerical solution and compare it with experimental measurements.

\section{Gas-Kinetic Method}

The present BGK scheme is similar to Xu's method, ${ }^{9,13}$ except with a simplification of the computation of the time evolution part. ${ }^{14}$ The scheme is briefly described as follows. First, the twodimensional BGK-Boltzmann equation is written as

$$
f_{t}+u f_{x}+v f_{y}=(g-f) / \tau
$$

where $f$ is the gas distribution function and $g$ is the equilibrium state approached by $f$. They are both functions of spaces $x$ and $y$, time $t$, particle velocities $(u, v)$, internal variable $\xi$, and $\tau$ is the particle collision time. The equilibrium state is assumed to be a Maxwellian distribution

$$
g=\rho(\lambda / \pi)^{(K+2) / 2} \exp \left\{-\lambda\left[(u-U)^{2}+(v-V)^{2}+\xi^{2}\right]\right\}
$$

where $\xi^{2}=\xi_{1}^{2}+\xi_{2}^{2}+\cdots+\xi_{K}^{2}$ represents the internal energy of particles, and $\lambda=\rho /(2 p)$. The total number of degrees of freedom $K$ in $\xi$ is equal to $(5-3 \gamma) /(\gamma-1)+1$ for a two-dimensional flow, which accounts for the independent rotational degrees of freedom and the random motion of particles in the $z$ direction. However, an equal temperature between the translational and rotational degree of freedom is assumed in the current model, and the bulk viscosity related to the rotational degree of freedom will appear automatically. During the particle collisions, $f$ and $g$ satisfy the conservation constraint

$$
\int(g-f) \psi \mathrm{d} \Xi=0
$$

at any point in space and time for the conservation of mass, momentum, and energy. Here $\mathrm{d} \Xi=\mathrm{d} u \mathrm{~d} v \mathrm{~d} \xi$ is the volume element in the phase space with $\mathrm{d} \xi=\mathrm{d} \xi_{1} \mathrm{~d} \xi_{2} \ldots \mathrm{d} \xi_{K}$, and $\psi$ is the vector of moments:

$$
\psi=\left(\psi_{1}, \psi_{2}, \psi_{3}, \psi_{4}\right)^{T}=\left[1, u, v,\left(u^{2}+v^{2}+\xi^{2}\right) / 2\right]^{T}
$$

Up to the Navier-Stokes order, the Chapman-Enskog expansion of the BGK model (1) at $(x=0, y=0, t=0)$ gives $f^{N S}=$ $g-\tau\left(g_{t}+u g_{x}+v g_{y}\right)$. Therefore, the gas distribution function 
around $(x=0, y=0)$ at the beginning of each time step $t=0$ up to the second-order accuracy can be approximated as

$$
f^{N S}=g+x g_{x}+y g_{y}-\tau\left(g_{t}+u g_{x}+v g_{y}\right)
$$

The preceding distribution function is used to reconstruct the initial condition around a cell interface. Then, the BGK equation is solved to get its time-dependent solution within a time step. The theoretical proof of the equivalence between the preceding expansion and the standard Chapman-Enskog expansion is presented in Ref. 18.

From Eqs. (1) and (3), the finite volume formulation of the BGK scheme is defined as

$$
\begin{aligned}
W_{i j}^{n+1} & =W_{i j}^{n}+\frac{1}{\Delta x} \int_{t^{n}}^{t^{n}+\Delta t}\left(F_{i-\frac{1}{2}, j}-F_{i+\frac{1}{2}, j}\right) \mathrm{d} t \\
& +\frac{1}{\Delta y} \int_{t^{n}}^{t^{n}+\Delta t}\left(G_{i, j-\frac{1}{2}}-G_{i, j+\frac{1}{2}}\right) \mathrm{d} t
\end{aligned}
$$

where $i$ and $j$ represent the cell numbers in the $x$ and $y$ directions, respectively. The relations between the distribution function $f$ and the macroscopic conservative quantities $W$ and their fluxes $F$ and $G$ are given by

$$
\begin{gathered}
W=(\rho, \rho U, \rho V, \rho \varepsilon)^{T}=\int f \psi \mathrm{d} \Xi \\
F=\int u f \psi \mathrm{d} \Xi, \quad G=\int v f \psi \mathrm{d} \Xi
\end{gathered}
$$

The BGK equation (1) has the integral solution

$$
\begin{gathered}
f(x, y, t, u, v, \xi)=\frac{1}{\tau} \int_{0}^{t} g\left(x^{\prime}, y^{\prime}, t^{\prime}, u, v, \xi\right) e^{-\left(t-t^{\prime}\right) / \tau} \mathrm{d} t^{\prime} \\
+\mathrm{e}^{-t / \tau} f_{0}(x-u t, y-v t, u, v, \psi)
\end{gathered}
$$

where $x^{\prime}=x-u\left(t-t^{\prime}\right), \quad y^{\prime}=y-v\left(t-t^{\prime}\right)$ is the trajectory of a particle motion and $f_{0}$ is the initial gas distribution function at the beginning of each time step $(t=0)$. The key of the BGK scheme is to construct $f_{0}$ and $g$ around the cell interface $(i+1 / 2, j)$,

$$
\begin{gathered}
f_{0}(x, y, u, v, \xi)=\left[1+a^{l} x+b^{l} y-\tau\left(a^{l} u+b^{l} v+A^{l}\right)\right](1-H[x]) g^{l} \\
+\left[1+a^{r} x+b^{r} y-\tau\left(a^{r} u+b^{r} v+A^{r}\right)\right] H[x] g^{r} \\
g(x, y, t, u, v, \xi)=\left[1+(1-H[x]) \bar{a}^{l} x+H[x] \bar{a}^{r} x+\bar{b} y+\bar{A} t\right] g_{0}
\end{gathered}
$$

where the initial gas distribution function at the left and right sides of the cell interface (denoted by superscripts $l$ and $r$ ) is constructed according to the Chapman-Enskog expansion [Eq. (5)]. The inclusion of discontinuity at a cell interface enhances the performance of the kinetic scheme in the capturing of flow discontinuities, such as the shocks, when the computational mesh size is not fine enough to resolve its physical structure. For convenience, in the local coordinate system $x$ and $y$ are assumed to be in the normal and tangential directions of a cell interface. Thus, the interface is described as $x_{i+1 / 2}=0$ and $-\Delta y / 2 \leq y \leq \Delta y / 2$. Here $H[x]$ is the Heaviside function of local coordinate $x$. In the preceding equations, $g^{l}, g^{r}$, and $g_{0}$ are local Maxwellians, which are obatined from the initial reconstructed conservative flow variables and their corresponding slopes. The local terms $a^{l}, a^{r}, \bar{a}^{l}, \bar{a}^{r}, b^{l}, b^{r}, \bar{b}, A^{l}, A^{r}$, and $\bar{A}$ are from the Taylor expansion of a Maxwellian and take the form

$$
\begin{array}{r}
a^{l}=a_{1}^{l}+a_{2}^{l} u+a_{3}^{l} v+a_{4}^{l}\left(u^{2}+v^{2}+\xi^{2}\right) / 2=a_{\alpha}^{l} \psi_{\alpha} \\
\alpha=1,2,3,4
\end{array}
$$

where all coefficients, $a_{\alpha}^{l}, \ldots, \bar{A}_{\alpha}$, are local constants. They are evaluated from the spatial and temporal slopes of the reconstructed conservative variables $W$, such as

$$
\begin{gathered}
\frac{\nabla_{x} W^{l}}{\rho^{l}}=\frac{1}{\rho^{l}} \int \psi_{\alpha} a^{l} g^{l} \mathrm{~d} \Xi=M_{\alpha \beta}^{l} a_{\beta}^{l}, \quad \frac{\nabla_{y} W^{l}}{\rho^{l}}=M_{\alpha \beta}^{l} b_{\beta}^{l} \\
M_{\alpha \beta}^{l} A_{\beta}^{l}=-\frac{1}{\rho^{l}} \int\left(a^{l} u+b^{l} v\right) g^{l} \psi_{\alpha} \mathrm{d} \Xi
\end{gathered}
$$

where the matrix $M_{\alpha \beta}^{l}$ is defined as

$$
M_{\alpha \beta}^{l}=\left(\frac{1}{\rho^{l}}\right) \int \psi_{\alpha} \psi_{\beta} g^{l} \mathrm{~d} \Xi
$$

The detail of the matrix and the solution of the preceding equations for all coefficients $a^{r}, b^{r}, \bar{b}, A^{r}$, and $\bar{A}$ are presented in our previous work. ${ }^{9,13,14}$ Here, it is emphasized that the inclusion of these coefficients $b^{l}, b^{r}, \bar{b}$ accounts for the effect of tangential variation of conservative variables at a cell interface. Different from shock-capturing schemes based on the Riemann solution, the current scheme simulates a multidimensional transport process across a cell interface.

The distribution function $f$ at a cell interface is evaluated from $f_{0}$ and $g$ through expression (8):

$$
\begin{aligned}
& f(0,0, t, u, v, \xi)=(1-C) g_{0}+[t+\tau(C-1)] \bar{A} g_{0} \\
& \quad+[t C+\tau(C-1)]\left[\bar{a}^{l} u H[u]+\bar{a}^{r} u(1-H[u])+\bar{b} v\right] g_{0} \\
& \quad+C\left\{\left[1-(t+\tau)\left(u a^{l}+v b^{l}\right)-\tau A^{l}\right] H[u] g^{l}\right. \\
& \left.\quad+\left[1-(t+\tau)\left(u a^{r}+v b^{r}\right)-\tau A^{r}\right](1-H[u]) g^{r}\right\}
\end{aligned}
$$

where $C$ is defined as $C=e^{-t / \tau}$. The fluxes across the cell interface can be calculated with Eq. (7), and the fluxes in the general coordinates can be obtained through coordinate transformation. The fluxes across the interface in the $j$ direction can be computed similarly. Finally, the conservative variables at the next time step can be calculated via the finite volume formulation (6). For the axisymmetric flow simulation, there is an additional source term in Eq. (6),

$$
S=\left[0,0, \Delta t\left(p-\tau_{33}\right) / r, 0\right]^{T}
$$

where $r$ is the distance from the cell center to the symmetry axis. The normal stress in the circumferential direction is

$\tau_{33}=2 \mu \varepsilon_{33}+(\varsigma-2 \mu / 3) \varepsilon_{i i}=-\frac{2}{5} \mu\left(\frac{\partial U}{\partial x}+\frac{\partial V}{\partial y}-\frac{4 V}{r}\right)$

Here, for a diatomic gas the bulk viscosity is $\varsigma=4 \mu / 15$. The splitting method is adopted to treat the preceding source term.

In Eq. (15), a simplified method ${ }^{14}$ is applied to calculate $\bar{A}$, which comes from the differentiation of the constraint at $(x=0, y=0, t=0)$,

$$
\left.\frac{\partial}{\partial t} \int(g-f) \psi_{\alpha} \mathrm{d} \Xi\right|_{x=0, y=0, t=0}=0
$$

This leads to

$$
M_{\alpha \beta}^{0} \bar{A}_{\beta}=\frac{1}{\rho_{0}} \int\left[A^{l} H[u] g^{l}+A^{r}(1-H[u]) g^{r}\right] \psi_{\alpha} \mathrm{d} \Xi
$$

where only two terms need to be calculated. This simplification leads to a decrease in CPU time.

The collision time $\tau$ is given by

$$
\tau=\mu_{0} / p_{0}+C_{1} \Delta t\left|p^{l}-p^{r}\right| /\left(p^{l}+p^{r}\right)
$$

where $\mu_{0}$ and $p_{0}$ are respectively the macroscopic viscosity coefficient and the pressure calculated at the cell interface. The last term 
in the preceding equation represents the dynamical artificial dissipation, and the constant $C_{1}$ can be chosen from 0 to 1 . The time step $\Delta t$ is calculated from the Courant-Friedrichs-Lewy condition. For turbulent flow, a turbulence model, such as for eddy viscosity, can be directly included through the collision time, which is similar to the continuum Navier-Stokes equations.

The present scheme is a second-order method in both space and time for a continuous flowfield. ${ }^{14,19}$ The accuracy of the scheme is however further ensured because of its multidimensional particle transport across a cell interface. The decrease of CPU time is attractive for the current scheme. However, because of many moments calculations the computational time of the current scheme for a typical two-dimensional flow problem is about 30\% greater than that required for Roe's flux difference splitting scheme with the inclusion of viscous flux. ${ }^{20}$ As can be judged from previous studies, the superior robustness and accuracy of the BGK scheme contribute to offset the burden of extra computational costs. Actually, for real engineering code the time spent on flux evaluation takes only a small amount of time in comparison with other computation, such as mesh generation. Another advantage of the BGK scheme is that the second-order accuracy in space and time can be extended up to the boundary by implementing different slopes of the flow quantities across the boundary according to the condition, such as isothermal and adiabatic conditions. It can also naturally introduce a slip condition through the control of reflecting particles in the near-continuum regime. Finally, for all viscous flow computations in the current paper a second-order interpolation based on the van Leer limiter for the conservative variables is used for the construction of the initial conditions of the gas distribution function at the beginning of each time step.

\section{Kinetic Wall Boundary Condition}

Many studies have simulated the gas flow in the near-continuum regime using the NS solver with slip boundary conditions. Because all computations in the BGK scheme are based on the particle distribution function, the slip boundary condition can be naturally constituted according to the interaction model between the gas and the solid boundary. ${ }^{15,16} \mathrm{~A}$ time-accurate gas distribution function near the wall $f^{i}$ can be obtained through a similar procedure as that for Eq. (15). With the one-sided interpolation of the conservative variables initially up to the wall, a time-accurate gas distribution function at the wall surface can be obtained:

$$
f^{i}=g_{0}[1-\tau(\bar{a} u+\bar{A})+t \bar{A}]
$$

in the normal direction, where the wall is assumed to be located at the left cell interface. Thus, the number of particles hitting the wall can be evaluated, and these particles will be reflected from the wall with a Maxwellian distribution constructed according to the wall temperature $\lambda_{w}$,

$$
g^{w}=\rho_{w}\left(\lambda_{w} / \pi\right)^{(K+2) / 2} \exp \left[-\lambda_{w}\left(u^{2}+v^{2}+\xi^{2}\right)\right]
$$

The requirement of no particles penetrating through the solid wall gives

$$
\int_{0}^{\Delta t} \int_{u<0} u f^{i} \mathrm{~d} \Xi \mathrm{d} t=-\int_{0}^{\Delta t} \int_{u>0} u g^{w} \mathrm{~d} \Xi \mathrm{d} t
$$

Then, the flow density $\rho_{w}$ in the preceding wall Maxwellian can be obtained:

$$
\rho_{w}=-\frac{2 \sqrt{\pi \lambda_{w}}}{\Delta t} \int_{0}^{\Delta t} \int_{u<0} u f^{i} \mathrm{~d} \Xi \mathrm{d} t
$$

Because the reflected particles might not fully accommodate the wall condition, an accommodation coefficient $\sigma$ is introduced to represent the diffusive reflection part of the particles and $(1-\sigma)$ to represent the specular reflection particles. Therefore, the final gas distribution function at the wall can be written as

$$
f^{t}=\sigma g_{u>0}^{w}+f_{u<0}^{i}+(1-\sigma) f_{u>0}^{i}(-u)
$$

where the last term accounts for the component with the specular reflection from the wall surface.

In high-speed flow, because of the relative movement of the flow to the solid wall, the temperature of the reflected particles can differ from the wall temperature. Thus, a thermal accommodation coefficient $\alpha$ can be introduced to represent the accommodation of the reflected particles with the wall:

$$
\alpha=\left(E_{i}-E_{r}\right) /\left(E_{i}-E_{w}\right)
$$

where $E_{i}$ is the total energy of the incident particles $(u<0), E_{r}$ is the reflected particles $(u>0)$, and $E_{w}$ is the particles with zero macrovelocity and wall temperature $(u>0)$,

$$
E_{w}=\rho_{w} / 4(\gamma-1) \lambda_{w}, \quad E_{r}=\rho_{r} / 4(\gamma-1) \lambda_{r}
$$

The coefficient with unit value, $\alpha=1$, represents the full thermal accommodation: particles are reflected with the wall temperature and Maxwellian velocity distribution. On the other hand, the coefficient with zero value, $\alpha=0$, indicates the adiabatic reflection of particles from the wall, that is, $E_{r}=E_{i}$.

Similar to Eq. (23), from the zero mass flux condition at the solid wall

$$
\int_{0}^{\Delta t} \int_{u<0} u f^{i} \mathrm{~d} \Xi \mathrm{d} t=-\int_{0}^{\Delta t} \int_{u>0} u g^{r} \mathrm{~d} \Xi \mathrm{d} t
$$

the relation between the reflecting particles and the scattering Maxwellian can be obtained:

$$
\rho_{r} / \sqrt{\lambda_{r}}=\rho_{w} / \sqrt{\lambda_{w}}
$$

From Eqs. (26), (27), and (29), the density and temperature of the reflected particles can be determined. Thus, the velocity distribution function at the solid wall can be constructed as

$$
f^{t}=g_{u>0}^{r}+f_{u<0}^{i}
$$

In brief, two kinetic boundary conditions are presented. One is a blend of diffusive and specular scattering, and a complete thermal accommodation is used for the diffusely scattered particles [Eq. (25)]. Another one has fully diffusive reflection but with partial thermal accommodation for those reflected particles according to the wall temperature [Eq. (30)]. Similarly, other kinds of kinetic boundary condition can be also constructed. Based on the preceding formulation, the flux across the solid boundary can be evaluated easily, and the slip boundary condition automatically forms in the BGK scheme. Note that for complex flow such as turbulence, the kinetic boundary condition in the near-continuum flow regime can give instantaneous values for the flow variables with good accuracy and simplicity in comparison with the slip boundary conditions used in continuum approaches.

\section{Results and Discussion}

The flow around a hollow cylinder flare is calculated in the present study. The configuration and the computational mesh are shown in Fig. 1 with the reference length $L=0.1017 \mathrm{~m}$. A stretched structural mesh with $N_{x} \times N_{y}$ cells in the $x$ and $y$ directions is adopted. Table 1 gives the computational parameters, where the symbol NB represents the calculation with the isothermal nonslip solid-wall boundary conditions, ${ }^{9}$ KB2 is the present kinetic wall boundary condition Eq. (30), and $\mathrm{KB}$ and $\mathrm{KB} 1$ are based on Eq. (25) with different accommodation coefficients.

The parameters of the freestream are given according to the experimental conditions: $p_{\infty}=6.3 \mathrm{~Pa}, T_{\infty}=51 \mathrm{~K}, M_{\infty}=9.91$, and $T_{w}=293 \mathrm{~K}$, which ensure a laminar flow condition for the entire configuration. The gas is nitrogen with $\mu=\mu_{\text {ref }}\left(T / T_{\text {ref }}\right)^{1.5}$ $\left(T_{\text {ref }}+T_{s}\right) /\left(T+T_{s}\right)$, with $\mu_{\text {ref }}=1.663 \times 10^{-5} \mathrm{~Pa} \cdot \mathrm{s}, \quad T_{\text {ref }}=$ $273.15 \mathrm{~K}, T_{s}=106.7 \mathrm{~K}$, the specific heat ratio $\gamma=1.4$, and the Prandtl number $\mathrm{Pr}=0.718$.

The linear extrapolation method is adopted for the top and outflow boundaries. The parameters of the inlet flow are set from the 
Table 1 Separation and reattachment point locations for different cases

\begin{tabular}{llccccr}
\hline \hline Case & $N_{x} \times N_{y}$ & Wall condition & $\Delta x_{\min }, \mathrm{mm}$ & $\Delta y_{\min }, \mathrm{mm}$ & $X_{\text {sep }} / L$ & $X_{\text {reat }} / L$ \\
\hline NB & $480 \times 160$ & Nonslip & 0.043 & 0.026 & 0.734 & 1.342 \\
NB1 & $240 \times 80$ & Nonslip & 0.086 & 0.053 & 0.755 & 1.332 \\
NB2 & $960 \times 320$ & Nonslip & 0.022 & 0.013 & 0.733 & 1.343 \\
KB & $480 \times 160$ & Kinetic $(\sigma=\alpha=1)$ & 0.043 & 0.026 & 0.743 & 1.336 \\
KB1 & $480 \times 160$ & Kinetic $(\sigma=0.5)$ & 0.043 & 0.026 & 0.805 & 1.305 \\
KB2 & $480 \times 160$ & Kinetic $(\alpha=0.5)$ & 0.043 & 0.026 & 0.762 & 1.340 \\
\hline \hline
\end{tabular}

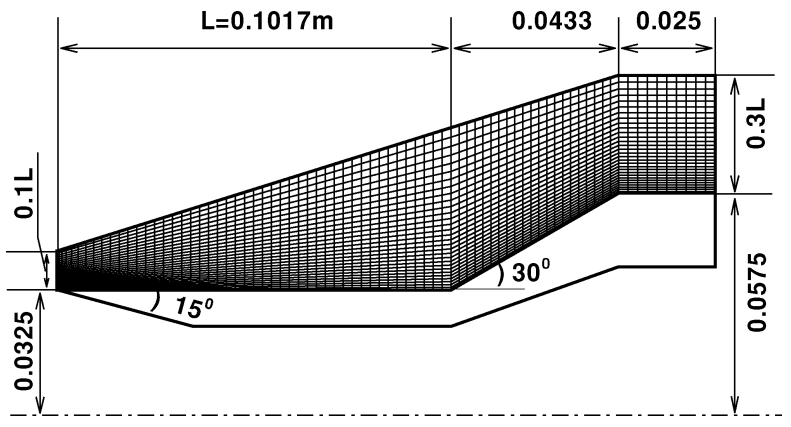

Fig. 1 Schematic view of the computational mesh.

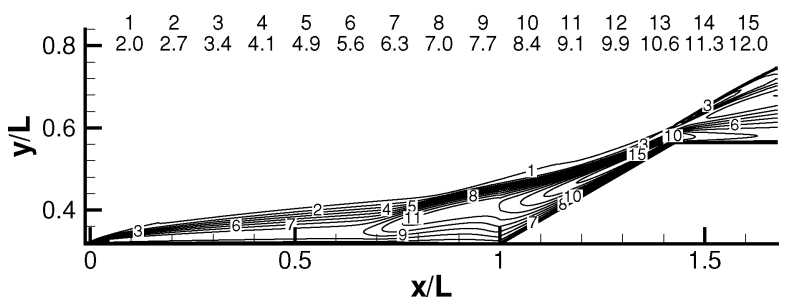

Fig. 2 Temperature contours $T / T_{\infty}$.

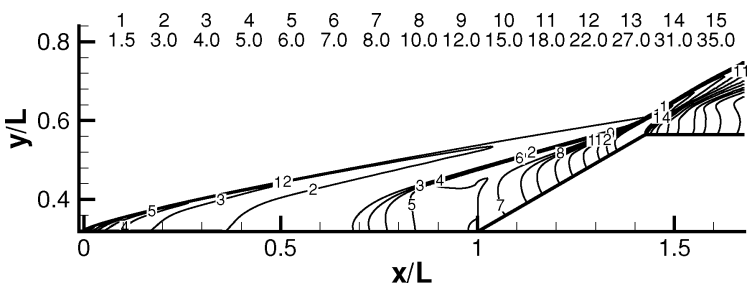

Fig. 3 Pressure contours $p / p_{\infty}$.

freestream. At the bottom boundary before the leading edge (about $N_{x} / 40$ cells), the symmetric reflection condition is used.

Figures 2 and 3 show the simulated temperature and pressure fields with the normal mesh (NB case in Table 1). All of the present calculations give the same flow pattern. A strong viscous interaction is observed at the leading edge of the hollow cylinder, forming the laminar boundary. The flow starts separating at $x / L>0.7$ and reattaches to the conical section near the point $x / L=1.3$. The separation shock wave, the reattachment shock waves, and the leadingedge shock wave interact with one another near the end of the conical part, resulting in the complex flow structure. The expansion waves occur over the cylindrical part downstream of the flare.

\section{Mesh Convergence}

To validate the results of the present study, computations with different grids are conducted. Figure 4 shows the pressure coefficient along the flare surface,

$$
C_{p}=\frac{p-p_{\infty}}{0.5 \rho_{\infty} u_{\infty}^{2}}
$$

compared with the experimental data. ${ }^{1}$ Good mesh convergence can be observed with the normal grid of $480 \times 160$ cells. It is the same

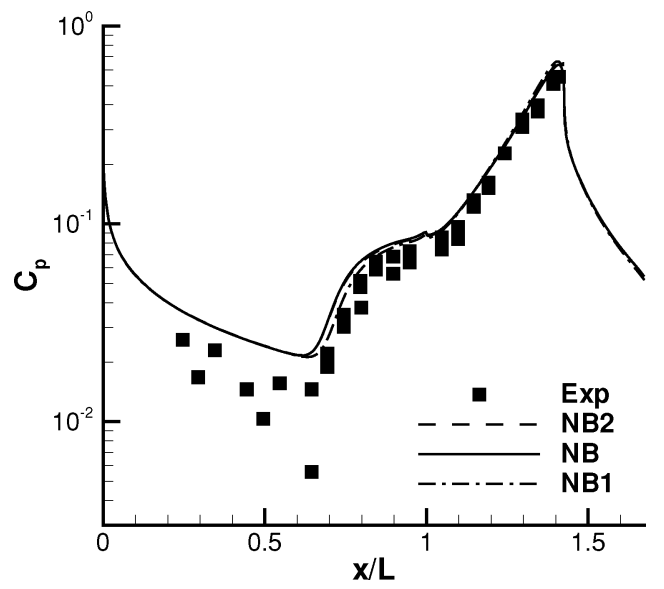

Fig. 4 Pressure coefficient along the flare surface using different meshes.

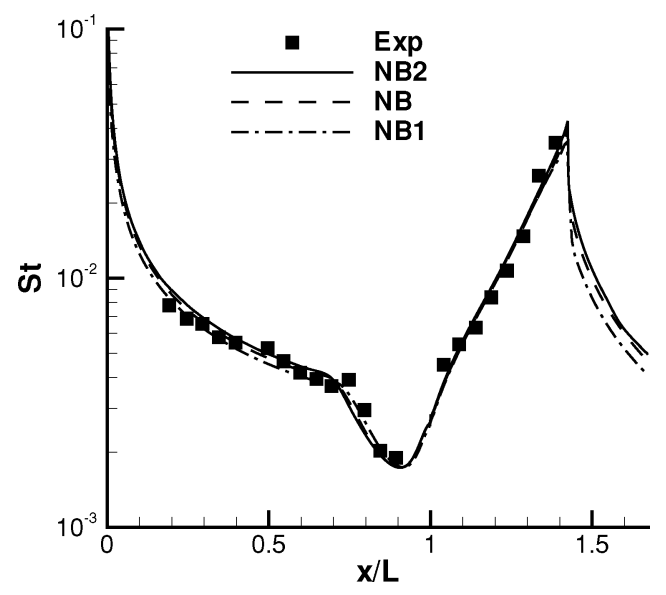

Fig. 5 Stanton number along the flare surface using different meshes.

for the wall Stanton number,

$$
S t=\frac{\left(\mu u_{\tau} \partial u_{\tau} / \partial n+\kappa \partial T / \partial n\right)_{w}}{\rho_{\infty} u_{\infty}\left(H_{0}-H_{w}\right)}
$$

where $H$ is the enthalpy, $\kappa$ is the thermal conductivity, and $u_{\tau}$ is the nonvanishing gas velocity on the wall (Fig. 5).

To validate the present results further, the length of the separation zone, which is very sensitive to cell size, is also studied. The recirculation zone length increases with grid refinement (shown in Table 1), which is in agreement with other numerical studies. ${ }^{2,17}$ The values $X_{\text {sep }} / L=0.734$ and $X_{\text {reat }} / L=1.342$ obtained with the normal mesh are nearly identical to those with the fine mesh (NB2 case), that is, $X_{\text {sep }} / L=0.733$ and $X_{\text {reat }} / L=1.343$, respectively. Again good mesh convergence is reached with the normal mesh.

\section{Computations with Nonslip Boundary Conditions Wall Quantities}

The present computed results under nonslip wall boundary conditions are compared with the experimental and DSMC data. ${ }^{1,2,17}$ 


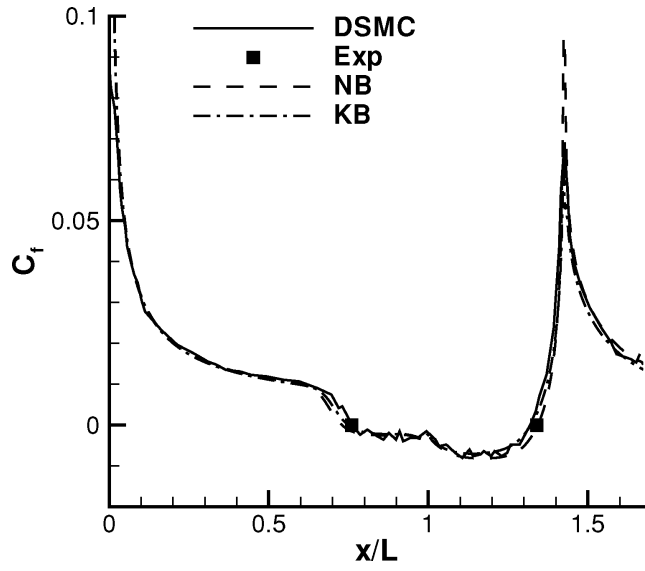

Fig. 6 Skin-friction coefficient along the flare surface using different numerical methods.

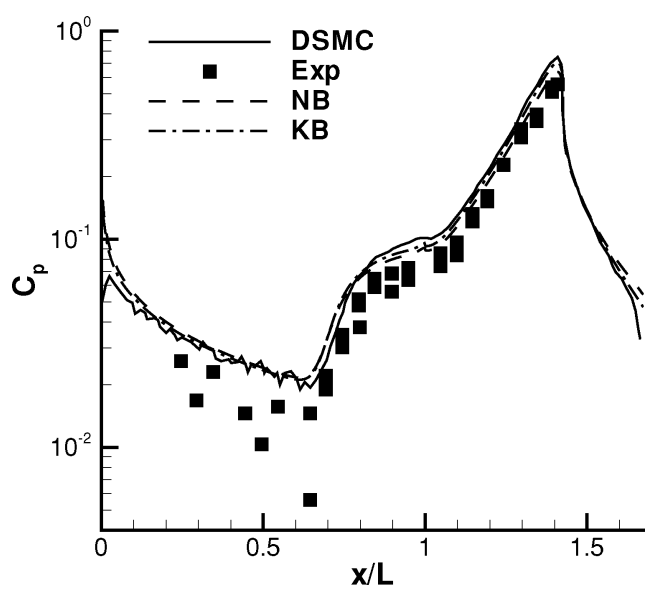

Fig. 7 Pressure coefficient distribution.

Figure 6 shows the wall skin-friction $C_{f}$ distribution, where the present results are observed to be very close to the experimental and DSMC results. The DSMC method predicts the separation location $X_{\text {sep }} / L=0.77$ (Ref. 17) better than the present BGK-NS solver with a nonslip wall boundary condition, which yields $X_{\mathrm{sep}} / L=0.734$, with respect to the experimental value $X_{\text {sep }} / L=0.76 \pm 0.01$. But the present calculated reattachment location $X_{\text {reat }} / L=1.342$ is closer to the experimental value $(1.34 \pm 0.01)$ than is the DSMC result (1.32). An overpredicted length of the separation zone via NS calculations was found in other studies, ${ }^{1,2,4,17}$ which can come from the rarefaction effects, especially near the leading edge of the flare, where slip wall boundary conditions are necessary.

Figure 7 shows the pressure coefficient distribution along the flare surface. The pressure decreases after the leading edge and begins to increases near $X / L=0.63$ as a result of the separation shock wave. In the recirculation region, the pressure follows a plateau distribution. Then, the reattachment shock wave causes the pressure increases until the end of the conical part of the flare where the expansion occurs. This typical structure of a shock/laminar boundarylayer interaction can also be observed in the wall skin-friction coefficient variation in Fig. 6. As in the DSMC and other NS studies, ${ }^{2,17}$ the present predicted pressure is higher than the measured results upstream of the cylinder. More experimental measurements might be required to resolve these discrepancies. ${ }^{1}$

The present predicted heating rate, shown in Fig. 8, from the Stanton number agrees quite well with the experimental data. The present results calculated from the BGK scheme seem better than those from the DSMC method, except for the greater peak value at the leading edge, where the DSMC can yield a better result. ${ }^{17}$ The heat flux decreases along the laminar boundary until the flow separation, which causes a rapid decrease and then an increase in the heating rate. The maximal heating rate is found downstream of

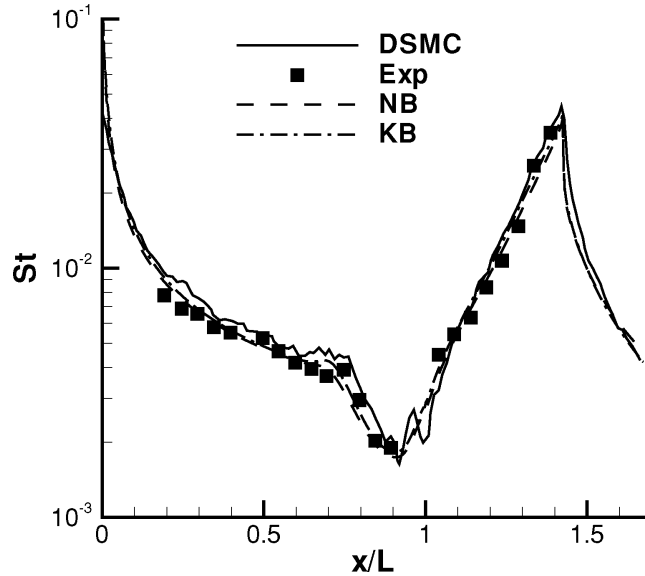

Fig. 8 Stanton-number distribution.

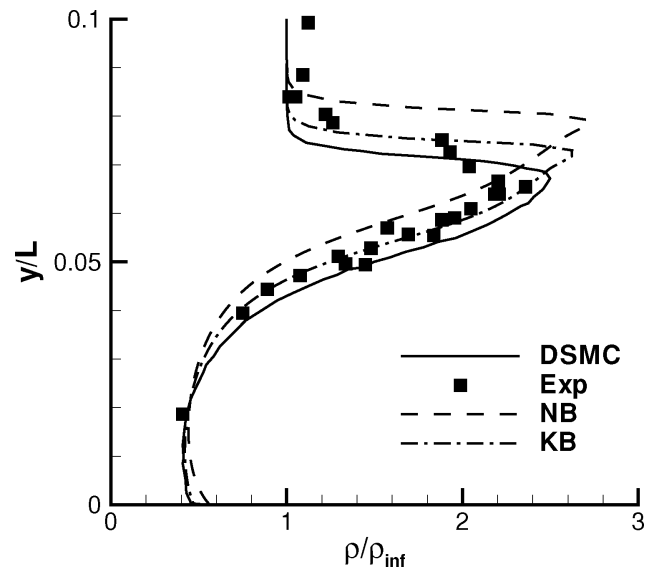

Fig. 9 Density profiles at $x / L=0.3$.

the reattachment point, just before the expansion into the cylindrical extension.

\section{Transverse Profiles}

Three transversal density profiles are compared with the measured data at different locations, $X / L=0.3,0.6$, and 0.76 . Figure 9 shows the profile at $X / L=0.3$, located ahead of the separation, where the leading shock wave causes an increase in the density. Good agreement can be seen between the numerical and experimental results for density peak amplitude, although the prediction of the radial shock position is not as good as with the DSMC method.

This discrepancy comes from the remaining influence of the leading-edge effects not accurately modeled by the NS solver, especially with the rarefaction effects not being taken into account, which affects the inclination of the leading-edge shock and results in the radial shock position deviations. The difference between the experimental leading edge and the numerically reconstructed one with a limited cell resolution can also contribute to this discrepancy. For profiles at $X / L=0.6$ and 0.76 , just around the flow separation, good agreement between the present calculations and the measurements is observed again as shown in Figs. 10 and 11. It is interesting that the present BGK-NS solver predicts the leading shock position better than does the DSMC method. The leading upstream shock inclination predicted by the NS solver is greater than the measured value.

\section{Effect of Slip Conditions}

As just discussed, the discrepancies between the present results, such as the separation zone length and the leading shock positions, obtained by the BGK-NS solver and the data from experiments or the DSMC method can come from the rarefaction effects, especially at the leading edge. The Knudsen number, based on the reference length $L$ and the freestream mean free path $\lambda_{\infty}, K n=\lambda_{\infty} / L$, 


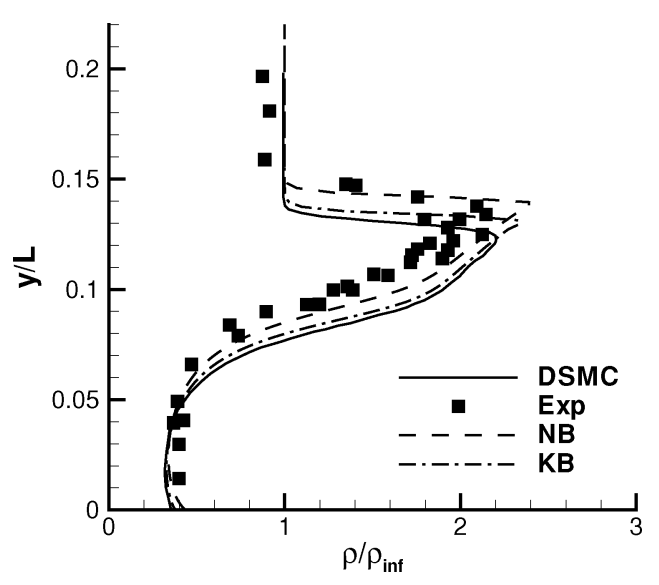

Fig. 10 Density profiles at $x / L=0.6$.

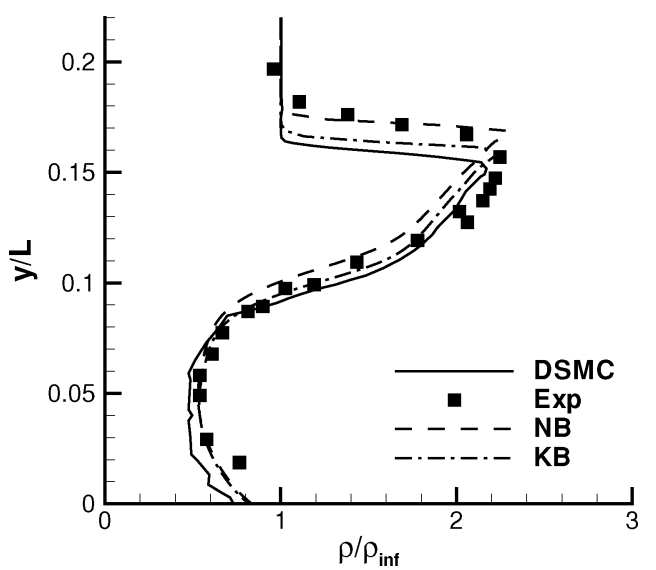

Fig. 11 Density profiles at $x / L=0.76$.

is usually used as the criterion of the rarefaction. The value is $K n=0.005$ for the present problem. Considering the variation of the mean free path in the flowfields, Graur et al. evaluated the local Knudsen number ${ }^{2}$ based on the density change along a streamline and found that the values in the vicinity of the leading edge and near the separation are larger than 0.01 . Thus, a slip boundary condition is required when simulating the current flow.

The present kinetic wall boundary conditions [Eqs. (21-30)] with accommodation coefficients, $\sigma=\alpha=1$ (KB case), are applied into the computations with the normal mesh. In general, the introduction of the slip boundary conditions by the kinetic conditions leads to results that are closer to the experimental measurements and DSMC predictions. The Stanton number (see Fig. 8) shows a decrease with the peak value at the leading edge and a slight increase in most of the flare surface until the end of the conical section. A little increase in the heat flux in the computational result before the separation can be observed in the figure with the slip condition, which is closer to the experimental data. On the other hand, the wall pressure, as shown in Fig. 7, exhibits a decrease along the flare surface before the flow separation and then a little increase until the flow expansion at downstream. The predicted separation point $X_{\text {sep }} / L=0.743$ (see Table 1 and Fig. 6) moves downstream, and the reattachment point $X_{\text {reat }} / L=1.336$ shows a slight movement upstream. Thus the computed separation zone length is shortened and agrees better with the experimental measurements.

As shown in Figs. 9-11, the density peaks calculated with the slip boundary conditions decrease slightly, and the leading shock-wave position moves evidently toward the flare, especially at the upstream location, $X / L=0.3$. Thus, the inclination of the leading shock decreases. These results are substantially closer to the measurement and DSMC calculation results. With the help of the slip boundary condition, the predicted density profiles are closer to the measured values than are the DSMC results, especially for the $X / L=0.76$ section.

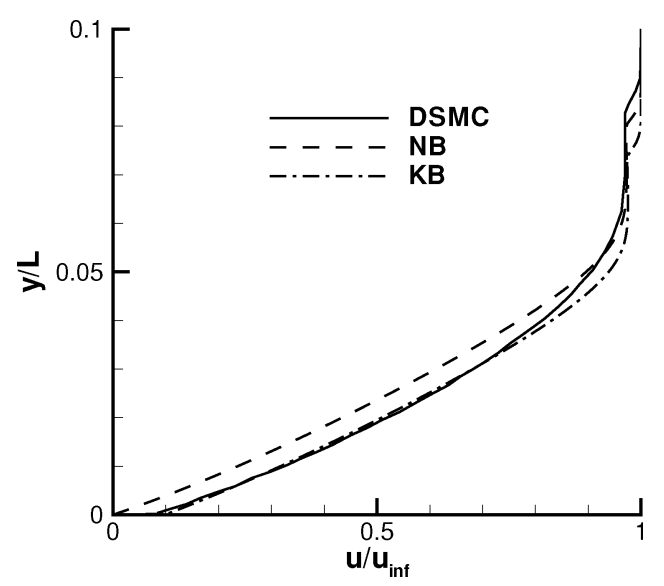

Fig. 12 Velocity profiles at $x / L=0.3$.

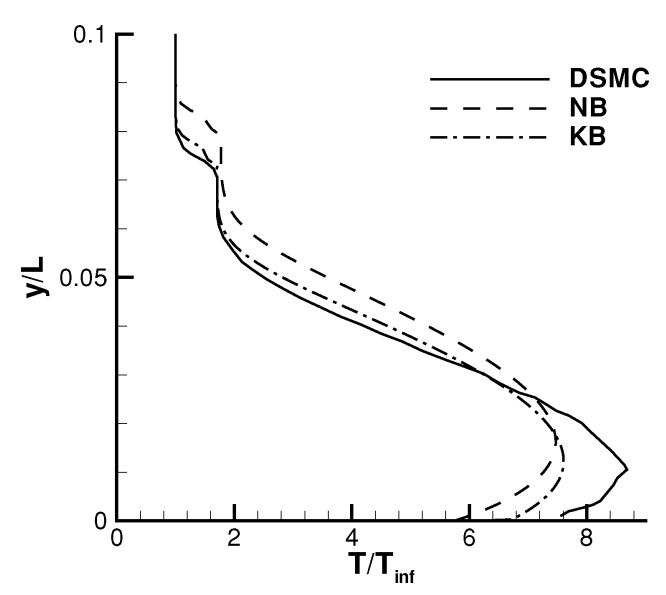

Fig. 13 Temperature profiles at $x / L=0.3$.

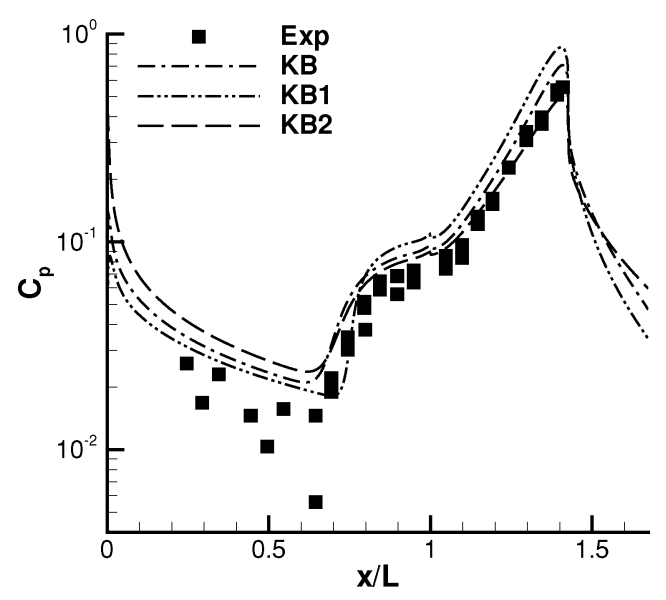

Fig. 14 Pressure coefficient distribution.

Consistent with the density distribution, the computed velocity and temperature profiles upstream with the kinetic boundary condition, shown in Figs. 12 and 13, are closer to the DSMC results. The implementation of the slip wall condition affects the flowfields not only near the wall, but also along the leading shock wave far away. The slip velocity near the wall is found to be about $10 \%$ of the freestream velocity, which cannot be neglected. This also confirms the necessity of using the slip wall boundary condition.

To investigate further the effect of kinetic boundary conditions, different accommodation coefficients are tested in the present study. As shown in Fig. 14, with $\sigma=0.5$ the computed separation point shifts downstream, while the reattachment point moves upstream. Thus, the recirculation zone length decreases evidently. In fact, the 


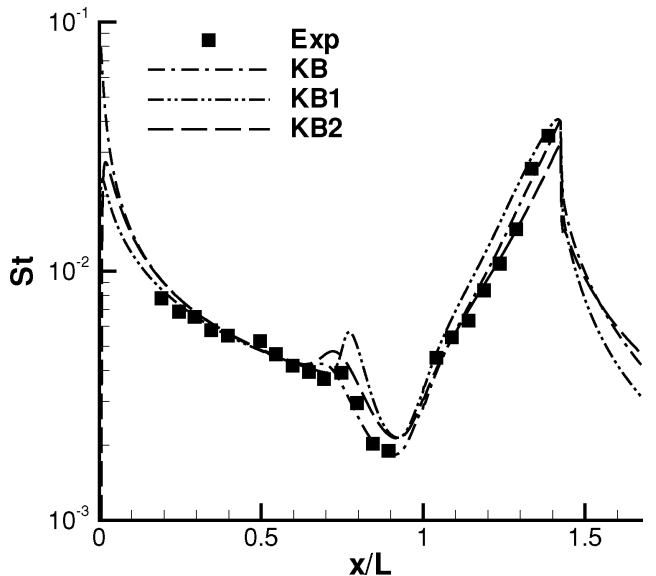

Fig. 15 Stanton-number distribution.

decrease in $\sigma$ means a decrease in the wall restriction on the stream flow, resulting in the decrease of the wall pressure before the separation and the suppression of the reverse pressure gradient. Hence, the separation point moves downstream. On the other hand, the change of the boundary conditions also affects the inclination of the leading-edge shock: the decrease in the wall restriction leads to a decrease in the shock-wave inclination, which causes a strong compression of the fluid in the cone region. As a result, the wall pressure increases, and the reattachment point moves upstream. Correspondingly, the wall heat flux reduces in the upstream regime, decreases downstream, and shows a distinct peak near the separation point (Fig. 15)

With the incomplete energy accommodation of the flow to the flare surface, such as $\alpha=0.5$, the present calculated separation point also moves downstream, and the reattachment point also shows a little shift downstream (see Table 1 and Figs. 14 and 15). The reduction of the thermal accommodation coefficient means a decrease in the heat exchange between the flow and the flare, except for the regime around the separation point. It also causes an increase in the internal energy of the flow and in the pressure, in the upstream region near the wall. The slope of the leading shock wave increases, and thus the pressure downstream decreases. The present computed trends of the wall quantities agree well with the DSMC result by Markelov et al. ${ }^{17}$ with the same energy accommodation coefficient.

The accommodation coefficients in the present kinetic boundary condition are set artificially. The reliable values should be determined by experiments despite the great difficulties of experimental setup. Nevertheless the present study shows the trends of the flowfield with variation in the accommodation coefficients and should be helpful in the evaluation of the physical coefficients. Besides this, there is a variable surface temperature $T_{w}$ in the experiments (especially near the leading edge). The equilibrium assumption between the translational and rotational temperatures of the gas can also contribute to the discrepancies in the present computational and the experimental results. ${ }^{17}$ It will be interesting to study these problems further.

\section{Conclusions}

The gas-kinetic BGK method with kinetic boundary conditions is described and applied to the numerical study of hypersonic flow past a hollow cylinder flare model. The computed results, including wall quantities and density profiles, are mesh convergent and agree well with results from experimental and DSMC studies. The application of the kinetic boundary conditions introduces the slip condition automatically and has substantial effects on the inclination of the leading shock wave and the wall quantities in some regions, such as those near the leading edge, the separation, and the reattachment points. With the kinetic boundary conditions, the predictions are closer to the DSMC computations and agree better with the experiments. The momentum/energy accommodation coefficients have a clear effect on the flare surface quantities and the leading-edge shock wave. The present study reveals the good performance of the BGK scheme in high-speed viscous flow simulations.

\section{Acknowledgments}

This work is supported by the National Natural Science Foundation of China through Grants 10302015 and 10232020 . Q. B. Li is also supported by China's Postdoctoral Science Foundation and K. Xu by the Hong Kong Research Grants Council (HKUST6116/03E and 6102/04E). We thank all of the reviewers for their helpful and constructive comments.

\section{References}

${ }^{1}$ Gorchakova, N., Kuznetsov, L., Yarygin, V., Chanetz, B., Pot, T., Bur, R., Taran, J. P., Pigache, D., Schulte, D., and Moss, J., "Progress in Hypersonic Studies Using Electron-Beam-Excited X-Ray Detection," AIAA Journal, Vol. 40, No. 4, 2002, pp. 593-598.

${ }^{2}$ Graur, I. A., Ivanov, M. S., Markelov, G. N., Burtschell, Y., Valerio, E., and Zeitoun, D., "Comparison of Kinetic and Continuum Approaches for Simulation of Shock Wave/Boundary Layer Interaction," Shock Waves, Vol. 12, No. 4, 2003, pp. 343-350.

${ }^{3}$ Wadhams, T. P., and Holden, M. S., "Summary of Experimental Studies for Code Validation in the LENS Facility and Comparisons with Recent Navier-Stokes and DSMC Solutions for Two- and Three-Dimensional Separated Regions in Hypervelocity Flows," AIAA Paper 2004-0917, Jan. 2004.

${ }^{4}$ Holden, M. S., Wadhams, T. P., Harvey, J. K., and Candler, G. V., "Comparisons Between Measurements in Regions of Laminar Shock Wave Boundary Layer Interaction in Hypersoinc Flow with Navier-Stokes and DSMC Solutions," AIAA Paper 2002-0435, Jan. 2002.

${ }^{5}$ Holden, M. S., and Wadhams, T. P., "Code Validation Study of Laminar Shock/Boundary Layer and Shock/Shock Interactions in Hypersonic Flow Part A: Experimental Measurements," AIAA Paper 2001-1031, Jan. 2001.

${ }^{6}$ Markelov, G. N., and Ivanov, M. S., "Kinetic Analysis of Hypersonic Laminar Separated Flows for Hollow Cylinder Flare Configurations," Journal of Spacecraft and Rockets, Vol. 38, No. 6, 2001, pp. 875-881.

${ }^{7}$ Chanetz, B., Benary, R., Bousquet, J. M., Bur, R., Pot, T., Grasso, F., and Moss, J., "Experimental and Numerical Study of the Laminar Separation in Hypersonic Flow," Aerospace Science and Technology, Vol. 2, No. 3, 1998, pp. 205-218.

${ }^{8}$ Ivanov, M. S., and Gimelshein, S. F., "Computational Hypersonic Rarefied Flows," Annual Review of Fluid Mechanics, Vol. 30, 1998, pp. 469-505.

${ }^{9} \mathrm{Xu}, \mathrm{K}$., "A Gas-Kinetic BGK Scheme for the Navier-Stokes Equations, and Its Connection with Artificial Dissipation and Godunov Method," Journal of Computational Physics, Vol. 171, No. 1, 2001, pp. 289-335.

${ }^{10} \mathrm{Li}$, Q. B., and Fu, S., "Numerical Simulation of High-Speed Planar Mixing Layer," Computers and Fluids, Vol. 32, No. 10, 2003, pp. 1357-1377.

${ }^{11} \mathrm{Li}$, Q. B., Chen, H., and Fu, S., "Large-Scale Vortices in High-Speed Mixing Layers," Physics of Fluids, Vol. 15, No. 10, 2003, pp. 3240-3244.

${ }^{12} \mathrm{Li}$, Q., "Numerical Study of Compressible Mixing Layer with BGK Scheme," Ph.D. Dissertation, Dept. of Engineering Mechanics, Tsinghua Univ., Beijing, April 2002.

${ }^{13} \mathrm{Xu}, \mathrm{K}$., Mao, M., and Tang, L., "A Multidimensional Gas-Kinetic BGK Scheme for Hypersonic Viscous Flow," Journal of Computational Physics, Vol. 203, No. 2, 2005, pp. 405-421.

${ }^{14} \mathrm{Li}$, Q. B., Fu, S., and Xu, K., "A Compressible Navier-Stokes Flow Solver with Scalar Transport," Journal of Computational Physics, Vol. 204, No. 2, 2005, pp. 692-714.

${ }^{15}$ Zhao, J., Li, Q. B., Zhang, G., and Fu, S., "Numerical Simulation of Gas Flow in Micro-Channels with BGK Scheme," Journal of Tsinghua University (Science and Technology), Vol. 43, No. 8, 2003, pp. 1083-1087.

${ }^{16} \mathrm{Xu}, \mathrm{K}$., and Li, Z., "Microchannel Flows in Slip Flow Regime: BGKBurnett Solutions," Journal of Fluid Mechanics, Vol. 513, 2004, pp. 87-110.

${ }^{17}$ Markelov, G. N., Kudryavtsev, A. N., and Ivanov, M. S., "Continuum and Kinetic Simulation of Laminar Separated Flow at Hypersonic Speeds," Journal of Spacecraft and Rockets, Vol. 37, No. 4, 2000, pp. 499-506.

${ }^{18}$ Ohwada, T., and Xu, K., "The Kinetic Scheme for the Full-Burnett Equations," Journal of Computational Physics, Vol. 201, No. 1, 2004, pp. 315-332.

${ }^{19}$ Ohwada, T., "On the Construction of Kinetic Schemes," Journal of Computational Physics, Vol. 177, No. 1, 2002, pp. 156-175.

${ }^{20}$ Chae, D. C., Kim, C., and Rho, O., "Development of an Improved GasKinetic BGK Scheme for Inviscid and Viscous Flows," Journal of Computational Physics, Vol. 158, No. 1, 2000, pp. 1-27.

G. Candler Associate Editor 